\title{
On varieties that are uniruled by lines
}

\author{
Andreas L. Knutsen, Carla Novelli and Alessandra Sarti
}

\begin{abstract}
Using the $\sharp$-minimal model program of uniruled varieties we show that, for any pair $(X, \mathcal{H})$ consisting of a reduced and irreducible variety $X$ of dimension $k \geqslant 3$ and a globally generated big line bundle $\mathcal{H}$ on $X$ with $d:=\mathcal{H}^{k}$ and $n:=h^{0}(X, \mathcal{H})-1$ such that $d<2(n-k)-4$, then $X$ is uniruled of $\mathcal{H}$-degree one, except if $(k, d, n)=(3,27,19)$ and a $\sharp$-minimal model of $(X, \mathcal{H})$ is $\left(\mathbb{P}^{3}, \mathcal{O}_{\mathbb{P}^{3}}(3)\right)$. We also show that the bound is optimal for threefolds.
\end{abstract}

\section{Introduction}

It is well known that an irreducible nondegenerate complex variety $X \subseteq \mathbb{P}^{n}$ of degree $d$ satisfies $d \geqslant n-\operatorname{dim} X+1$. Varieties for which equality is obtained are the well-known varieties of minimal degree, which have been completely classified.

Varieties with $d$ 'small' compared to $n$ have been the objects of intensive study over the years; see e.g. [Har74, Ba75, Fuj77, Fuj75, Fuj80, Isk77, Ion85, Hor83, Rei86, Mel02]. One of the common features is that such varieties are covered by rational curves.

More generally one can study pairs $(X, \mathcal{H})$ where $X$ is an irreducible $k$-dimensional variety (possibly with some additional assumptions on its singularities) and $\mathcal{H}$ a line bundle on $X$ that is sufficiently 'positive' (e.g. ample or (birationally) very ample or big and nef). Naturally we set $d:=\mathcal{H}^{k}$ and $n:=\operatorname{dim}|\mathcal{H}|$. The difference between $d$ and $n$ is measured by the $\Delta$-genus: $\Delta(X, \mathcal{H}):=d+k-n-1$, introduced by Fujita (cf. [Fuj77] and [Fuj75]), who in fact shows that $\Delta(X, \mathcal{H}) \geqslant 0$ for $X$ smooth and $\mathcal{H}$ ample and that $\mathcal{H}$ is very ample if equality holds, so that the cases with $\Delta(X, \mathcal{H})=0$ are the varieties of minimal degree. The cases with $\Delta(X, \mathcal{H})=1$ have been classified by Fujita [Fuj80, Fuj81, Fuj84] and Iskovskih [Isk77].

If $\mathcal{H}$ is globally generated we can consider the morphism $\varphi_{\mathcal{H}}: X \rightarrow X^{\prime} \subseteq \mathbb{P}^{n}$ defined by $|\mathcal{H}|$. One has $d=\left(\operatorname{deg} \varphi_{\mathcal{H}}\right)\left(\operatorname{deg} X^{\prime}\right)$ and $\operatorname{deg} X^{\prime} \geqslant n-k+1$. If $d<2(n-k)+2$ the morphism $\varphi_{\mathcal{H}}$ is forced to be birational and $\operatorname{deg} X^{\prime}=d$. Hence in the range $d<2(n-k)+2$ studying nondegenerate degree $d$ varieties in $\mathbb{P}^{n}$, or pairs $(X, \mathcal{H})$ with $\mathcal{H}$ globally generated and big, is equivalent. Moreover, as the property of being globally generated and big is preserved from $\mathcal{H}$ to $f^{*} \mathcal{H}$ under a resolution of singularities $f$, this approach is suitable also to study singular varieties.

The notion of being covered by rational curves is incorporated in the concept of a variety being uniruled: a variety is uniruled if through any point there passes a rational curve. With the notation above, $d<k(n-k)+2$ is an optimal bound for uniruledness by [Mel02, Theorem A.3 and Example A.4].

In many ways uniruled varieties are the natural generalization to higher dimensions of ruled surfaces. In the Mori program they play an important role, because - as in the case of ruled surfaces these are the varieties for which the program does not yield a minimal model, but a Mori fiber space.

Received 1 March 2005, accepted in final form 23 January 2006.

2000 Mathematics Subject Classification 14E30, 14J30, 14J40, 14N25 (primary), 14C20, 14H45 (secondary).

Keywords: minimal model program, rational curves, 3 -folds, $n$-folds, linear systems.

This journal is (c) Foundation Compositio Mathematica 2006. 


\section{A. L. Knutsen, C. Novelli And A. Sarti}

Uniruled varieties can also be considered to be the natural generalizations to higher dimensions of surfaces of negative Kodaira dimension: in fact it is conjectured that a (smooth) variety is uniruled if and only if its Kodaira dimension is negative. The conjecture has been established for threefolds by Miyaoka [Miy88].

With the evolution of a structure theory for higher dimensional varieties in the past decades, namely the Mori program, the geometry of rational curves on varieties has gained new importance. The main idea is to obtain information about varieties by studying the rational curves on them (cf. e.g. [Kol96]).

To measure the 'degree' of the rational curves that cover $X$ we say in addition that $X$ is uniruled of $\mathcal{H}$-degree at most $m$ if the covering curves all satisfy $\Gamma \cdot \mathcal{H} \leqslant m$. Returning to the case where $\mathcal{H}$ is globally generated and the morphism $\varphi_{\mathcal{H}}: X \rightarrow X^{\prime} \subseteq \mathbb{P}^{n}$ is birational as above, we see that $X$ is uniruled of $\mathcal{H}$-degree at most $m$ if and only if $X^{\prime}$ is covered by rational curves of degrees at most $m$.

For surfaces Xiao [Xia86] and Reid [Rei86] independently found bounds on the uniruledness degree of $(X, \mathcal{H})$ depending on $d$ and $n$. For instance they showed that an irreducible, nondegenerate surface $X \subseteq \mathbb{P}^{n}$ is uniruled by lines if $d<\frac{4}{3}(n-2)$, except when $n=9$ and $\left(X, \mathcal{O}_{X}(1)\right)=$ $\left(\mathbb{P}^{2}, \mathcal{O}_{\mathbb{P}^{2}}(3)\right)$. The same result was obtained by Horowitz [Hor83] using a different approach. In particular, it immediately follows (by taking surface sections and using that $\left(\mathbb{P}^{2}, \mathcal{O}_{\mathbb{P}^{2}}(3)\right.$ ) cannot be a hyperplane section of any threefold other than a cone) that an irreducible, nondegenerate $k$-dimensional variety $X \subseteq \mathbb{P}^{n}$ is uniruled by lines for $k \geqslant 3$ if $d<\frac{4}{3}(n-k)$. (Note that if one assumes $X$ smooth, one gets the better bound $d<\frac{3}{2}(n-k-1)$, since $X$ is ruled by planes or quadrics in this range by [Hor83, Corollary p. 668].) However it is to be expected that this 'naive' inductive procedure does not yield an optimal bound.

The purpose of this paper is to obtain a bound for uniruledness degree one which is optimal for threefolds and independent of singularities. In fact we show the following result.

Theorem 0.1. Let $(X, \mathcal{H})$ be a pair consisting of a reduced and irreducible three-dimensional variety $X$ and a globally generated big line bundle $\mathcal{H}$ on $X$. Set $d:=\mathcal{H}^{3}$ and $n:=h^{0}(X, \mathcal{H})-1$.

If $d<2 n-10$ then $X$ is uniruled of $\mathcal{H}$-degree one, except when $(d, n)=(27,19)$ and a $\sharp$-minimal model of $(X, \mathcal{H})$ is $\left(\mathbb{P}^{3}, \mathcal{O}_{\mathbb{P}^{3}}(3)\right)$.

(For the definition of a $\sharp$-minimal model we refer to Definition 1.4 below.)

The bound in Theorem 0.1 is sharp, since there are pairs satisfying $d=2 n-10$ for infinitely many $d$ and $n$, namely $\left(\mathbb{P}^{2} \times \mathbb{P}^{1}, \mathcal{O}_{\mathbb{P}^{2}}(2) \otimes \mathcal{O}_{\mathbb{P}^{1}}(a)\right)$ for $a \geqslant 2$ (cf. Example 2.5 below), which are not uniruled of $\mathcal{H}$-degree one.

Observe that in Remark 2.3 below we obtain a better bound than in Theorem 0.1 for $8 \leqslant n \leqslant 12$.

As a consequence of Theorem 0.1 we get the following result for higher dimensional varieties, which is probably far from being sharp.

Corollary 0.2. Let $(X, \mathcal{H})$ be a pair consisting of a reduced and irreducible $k$-dimensional variety $X, k \geqslant 4$, and a globally generated big line bundle $\mathcal{H}$ on $X$. Set $d:=\mathcal{H}^{k}$ and $n:=h^{0}(X, \mathcal{H})-1$. If $d<2(n-k)-4$ then $X$ is uniruled of $\mathcal{H}$-degree one.

For those preferring the notion of $\Delta$-genus, the condition $d<2(n-k)-4$ is equivalent to $\Delta(X, \mathcal{H})<n-k-5=h^{0}(X, \mathcal{H})-\operatorname{dim} X-6$.

The above results have the following corollary for embedded varieties.

Corollary 0.3. Let $X \subset \mathbb{P}^{n}$ be a nondegenerate reduced and irreducible variety of dimension $k \geqslant 3$ and degree $d$. If $d<2(n-k)-4$ then $X$ is uniruled by lines, except when $(k, d, n)=(3,27,19)$ and a $\sharp$-minimal model of $\left(X, \mathcal{O}_{X}(1)\right)$ is $\left(\mathbb{P}^{3}, \mathcal{O}_{\mathbb{P}^{3}}(3)\right)$. 


\section{ON VARIETIES THAT ARE UNIRULED BY LINES}

Note that the condition $d<2(n-k)-4$ implicitly requires $n \geqslant k+6$ in the three results above.

To prove these results we use the $\sharp$-minimal model program of uniruled varieties introduced for surfaces by Reid in [Rei86] and developed for threefolds by Mella in [Mel02]. The main advantage of the $\sharp$-minimal model program is that one does not only work with birational modifications along the minimal model program but also uses a polarizing divisor. Under certain assumptions one manages to follow every step of the program on an effective divisor, i.e. a (smooth) surface in the case of threefolds.

Our method of proof uses the classification results in [Mel02] and borrows ideas from [Rei86]. The crucial point is a careful investigation of pairs $(X, \mathcal{H})$ such that the output of the $\sharp$-minimal model program is a particular type of Mori fiber space, which we call a terminal Veronese fibration (see Definition 3.1 below). This is roughly speaking a terminal threefold marked by a line bundle with at most base points fibered over a smooth curve with general fibers being smooth Veronese surfaces (with respect to the marking line bundle) and having at most finitely many fibers being cones over a smooth quartic curve. We find a lower bound on the degree of such a threefold (in fact on every marked terminal threefold having a terminal Veronese fibration as a $\sharp$-minimal model) and on the number of degenerate fibers of the members of the marking linear system.

The precise statement, which we hope might be of independent interest, is the following one.

Proposition 0.4. Let $(X, \mathcal{H})$ be a three-dimensional terminal Veronese fibration (see Definition 3.1) over a smooth curve $B$ and set $n:=h^{0}(\mathcal{H})-1$ and $d:=\mathcal{H}^{3}$. Then $d \geqslant 2 n-10$ and the general member of $|\mathcal{H}|$ is a smooth surface fibered over $B$ with at least $\frac{1}{2}(n-5)$ fibers that are unions of two conics (with respect to $\mathcal{H}$ ) intersecting in one point (the other fibers are smooth quartics).

Observe that both equalities are obtained by $\left(\mathbb{P}^{2} \times \mathbb{P}^{1}, \mathcal{O}_{\mathbb{P}^{2}}(2) \otimes \mathcal{O}_{\mathbb{P}^{1}}(a)\right)$; cf. Examples 2.5 and 3.4 below.

In $\S 1$ we set notation and give all central definitions. Moreover we introduce, after [Mel02], the $\sharp$-minimal models of pairs $(X, \mathcal{H})$ where $X$ is a terminal, $\mathbb{Q}$-factorial threefold and $\mathcal{H} \in \operatorname{Pic} X$ such that the general element in $|\mathcal{H}|$ is a smooth surface of negative Kodaira dimension (Theorem 1.2) and obtain results that are essential for the rest of the paper in Lemmas 1.1 and 1.5.

In $\S 2$ we first obtain an 'easy bound' on $d$ such that a threefold is uniruled in degree one (Proposition 2.1) and then we show how to reduce the proofs of our main results (Theorem 0.1 and its two corollaries) to a result about uniruled threefolds having a terminal Veronese fibration as a $\sharp$-minimal model, namely Proposition 2.4.

The proofs of Proposition 2.4 and of Proposition 0.4 are then settled in $\S 3$.

Finally, in $\S 4$ we give some final remarks, including a slight improvement of a result in [Mel02] and of Theorem 0.1 and Corollary 0.2.

\section{1. $\sharp$-minimal models of uniruled threefolds}

We work over the field of complex numbers.

A reduced and irreducible three-dimensional variety will be called a threefold, for short.

A $k$-dimensional projective variety $X$ is called uniruled if there is a variety $Y$ of dimension $k-1$ and a generically finite dominant rational map $p: Y \times \mathbb{P}^{1}-->X$. In particular, such a variety is covered by rational curves (cf. [Kol96, ch. IV, Corollary 1.4.4]).

If $\mathcal{H}$ is a nef line bundle on $X$ and $m \in \mathbb{Q}$ we say that $X$ is uniruled of $\mathcal{H}$-degree at most $m$ if $\operatorname{deg}\left(p^{*} \mathcal{H}\right)_{\mid \mathbb{P}^{1} \times\{y\}} \leqslant m$ for every $y \in Y$, or equivalently if there is a dense open subset $U \subseteq X$ such that every point in $U$ is contained in a rational curve $C$ with $C \cdot \mathcal{H} \leqslant m$ (cf. [Kol96, ch. IV, Proposition-Definition 1.4.]). A consequence is that in fact every point in $X$ is contained in a rational 


\section{A. L. Knutsen, C. Novelli and A. Sarti}

curve $C$ with $C \cdot \mathcal{H} \leqslant m$ (cf. [Kol96, ch. IV, Corollary 1.4.4]). In particular, if $X \subseteq \mathbb{P}^{n}$ we say that $X$ is uniruled by lines if $m=1$ with respect to $\mathcal{H}:=\mathcal{O}_{X}(1)$.

For a pair $(X, \mathcal{H})$ where $X$ is terminal $\mathbb{Q}$-factorial and $\mathcal{H}$ is a line bundle on $X$ with $|\mathcal{H}| \neq \emptyset$, the threshold of the pair is defined as

$$
\begin{aligned}
\rho(X, \mathcal{H}):=\sup \left\{m \in \mathbb{Q}: r m K_{X}\right. \text { is Cartier and } \\
\left.\qquad\left|r\left(\mathcal{H}+m K_{X}\right)\right| \neq \emptyset \text { for some } r \in \mathbb{Z}_{>0}\right\} \geqslant 0
\end{aligned}
$$

(cf. [Rei86, equation (2.1)] and [Mel02, Definition 3.1]).

Moreover we set

$$
d(X, \mathcal{H}):=\mathcal{H}^{\operatorname{dim} X} \quad \text { and } \quad n(X, \mathcal{H}):=h^{0}(X, \mathcal{H})-1=\operatorname{dim}|\mathcal{H}| .
$$

In these terms the $\Delta$-genus, introduced by Fujita (cf. [Fuj77] and [Fuj75]), is

$$
\Delta(X, \mathcal{H}):=d(X, \mathcal{H})+\operatorname{dim} X-n(X, \mathcal{H})-1,
$$

and all the results in this paper can be equivalently formulated with the $\Delta$-genus.

Recall that a surjective morphism $f: X \rightarrow Y$ with connected fibers between normal varieties is called a Mori fiber space if $-K_{X}$ is $f$-ample, $\operatorname{rk} \operatorname{Pic}(X / Y)=1$ and $\operatorname{dim} X>\operatorname{dim} Y$.

The following easy consequence of Clifford's theorem will be useful for our purposes.

Lemma 1.1. Let $(X, \mathcal{H})$ be a pair with $X$ a terminal $\mathbb{Q}$-factorial threefold and $\mathcal{H}$ a globally generated and big line bundle on $X$. Set $d:=d(X, \mathcal{H})$ and $n:=n(X, \mathcal{H})$. If $d<2 n-4$, then the following hold:

(i) The general surface $S \in|\mathcal{H}|$ is smooth with negative Kodaira dimension. In particular $X$ is uniruled and $\rho(X, \mathcal{H})<1$.

(ii) For any smooth irreducible $S \in|\mathcal{H}|$ and for any irreducible curve $D \in\left|\mathcal{H}_{\mid S}\right|$ we have

$$
D \cdot K_{S} \leqslant d-2 n+2 .
$$

Proof. The general element $S \in|\mathcal{H}|$ is a smooth irreducible surface by Bertini's theorem, as $X$ has isolated singularities (cf. [Mel02, §2.3]).

Pick any irreducible curve $D \in\left|\mathcal{O}_{S}(\mathcal{H})\right|$. Then $\operatorname{deg} \mathcal{O}_{D}(\mathcal{H})=\mathcal{H}^{3}=d$ and from

$$
0 \longrightarrow \mathcal{O}_{X} \longrightarrow \mathcal{H} \longrightarrow \mathcal{O}_{S}(\mathcal{H}) \longrightarrow 0
$$

and

$$
0 \longrightarrow \mathcal{O}_{S} \longrightarrow \mathcal{O}_{S}(\mathcal{H}) \longrightarrow \mathcal{O}_{D}(\mathcal{H}) \longrightarrow 0
$$

we get

$$
h^{0}\left(\mathcal{O}_{D}(\mathcal{H})\right) \geqslant h^{0}\left(\mathcal{O}_{S}(\mathcal{H})\right)-1 \geqslant h^{0}(\mathcal{H})-2=n-1
$$

Hence

$$
\operatorname{deg} \mathcal{O}_{D}(\mathcal{H})-2\left(h^{0}\left(\mathcal{O}_{D}(\mathcal{H})\right)-1\right) \leqslant d-2(n-2)<0,
$$

whence by Clifford's theorem on irreducible singular curves (see the appendix of [EKS88]) we must have $h^{1}\left(\mathcal{O}_{D}(\mathcal{H})\right)=0$, so that $\chi\left(\mathcal{O}_{D}(\mathcal{H})\right)=h^{0}\left(\mathcal{O}_{D}(\mathcal{H})\right) \geqslant n-1$. From $(5)$ we get

$$
\chi\left(\mathcal{O}_{S}(D)\right)-\chi\left(\mathcal{O}_{S}\right)=\chi\left(\mathcal{O}_{D}(\mathcal{H})\right)=h^{0}\left(\mathcal{O}_{D}(\mathcal{H})\right) \geqslant n-1 .
$$

Combining with Riemann-Roch we get

$$
\begin{aligned}
D \cdot K_{S} & =D^{2}-2\left(\chi\left(\mathcal{O}_{S}(D)\right)-\chi\left(\mathcal{O}_{S}\right)\right) \\
& \leqslant d-2 n+2<-2
\end{aligned}
$$




\section{ON VARIETIES THAT ARE UNIRULED BY LINES}

proving part (ii) and showing that $\kappa(S)<0$. (The latter fact also follows from [Mel02, Theorem A.3].) Now the fact that $X$ is uniruled with $\rho(X, \mathcal{H})<1$ follows from [Mel02, Definition 5.1 and Lemma 5.2].

In the following theorem we collect all the results of Mella [Mel02] that will be useful to us.

Theorem 1.2. Let $(X, \mathcal{H})$ be a pair with $X$ a terminal $\mathbb{Q}$-factorial threefold and $\mathcal{H}$ a globally generated and big line bundle on $X$, such that the general element in $|\mathcal{H}|$ is a smooth surface of negative Kodaira dimension.

Then there exist a pair $\left(X^{\sharp}, \mathcal{H}^{\sharp}\right)$ and a birational map $\phi: X--\rightarrow X^{\sharp}$ such that the following hold.

(i) $X^{\sharp}$ is terminal and $\mathbb{Q}$-factorial, $\mathcal{H}^{\sharp} \in \operatorname{Pic} X^{\sharp},\left|\mathcal{H}^{\sharp}\right|$ has at most base points, and $\rho\left(X^{\sharp}, \mathcal{H}^{\sharp}\right)=$ $\rho(X, \mathcal{H})=: \rho$.

(ii) Map $\phi$ is a finite composition of Mori extremal contractions and flips, and $\rho K_{X^{\sharp}}+\mathcal{H}^{\sharp}$ is $\mathbb{Q}$-nef.

(iii) For any smooth irreducible $S \in|\mathcal{H}|, f:=\phi_{\mid S}$ is a birational morphism, and $S^{\sharp}:=f(S)$ is a smooth surface in $\left|\mathcal{H}^{\sharp}\right|$.

(iv) If $X^{\sharp}$ is uniruled of $\mathcal{H}^{\sharp}$-degree at most $m$, then $X$ is uniruled of $\mathcal{H}$-degree at most $m$.

(v) $\left(X^{\sharp}, \mathcal{H}^{\sharp}\right)$ belongs to the following list:

(I) a $\mathbb{Q}$-Fano threefold with $K_{T^{\sharp}} \sim-(1 / \rho) \mathcal{H}^{\sharp}$, belonging to Table 1;

(II) a bundle over a smooth curve with generic fiber $\left(F, \mathcal{H}_{\mid F}^{\sharp}\right) \cong\left(\mathbb{P}^{2}, \mathcal{O}_{\mathbb{P}^{2}}(2)\right)$ and with at most finitely many fibers $\left(G, \mathcal{H}_{\mid G}^{\sharp}\right) \cong\left(\mathbf{S}_{4}, \mathcal{O}_{\mathbf{S}_{4}}(1)\right)$, where $\mathbf{S}_{4} \subset \mathbb{P}^{5}$ is the cone over the normal quartic curve $(\rho=2 / 3)$;

(III) a quadric bundle with at most $c A_{1}$ singularities and $\mathcal{H}_{\mid F}^{\sharp} \sim \mathcal{O}_{F}(1)$ for every fiber $F$ $(\rho=1 / 2)$;

(IV) $(\mathbb{P}(\mathcal{E}), \mathcal{O}(1))$ where $\mathcal{E}$ is a rank 3 vector bundle over a smooth curve $(\rho=1 / 3)$;

$(\mathrm{V})(\mathbb{P}(\mathcal{E}), \mathcal{O}(1))$ where $\mathcal{E}$ is a rank 2 vector bundle over a surface of negative Kodaira dimension $(\rho=1 / 2)$.

Proof. By [Mel02, Definition 5.1 and Lemma 5.2] we have $\rho(X, \mathcal{H})<1$. Now the existence of a pair $\left(X^{\sharp}, \mathcal{H}^{\sharp}\right)$ and a map satisfying conditions (i)-(iii) follows combining [Mel02, Theorem 3.2, Proposition 3.6 and Corollary 3.10] observing that it is implicitly shown in the proof of [Mel02, Theorem 3.2] that $\rho\left(X^{\sharp}, \mathcal{H}^{\sharp}\right)=\rho(X, \mathcal{H})$.

Property (v) follows from [Mel02, Theorem 5.3 and Definition 5.1], noting that the values of $\rho$ are explicitly given in each of the cases in the course of the proof of [Mel02, Theorem 5.3].

We have left to prove (iv). By assumption $X^{\sharp}$ is covered by a family of rational curves $\{\Gamma\}$ such that $\Gamma \cdot \mathcal{H}^{\sharp} \leqslant m$. The strict transform $\tilde{\Gamma}$ on $X$ of each such $\Gamma$ then satisfies $\tilde{\Gamma} \cdot S \leqslant m$ by [Mel02, Lemma 3.15].

In the cases (I) the general $S^{\sharp} \in\left|\mathcal{H}^{\sharp}\right|$ is a smooth del Pezzo surface and $\mathcal{O}_{S^{\sharp}}\left(\mathcal{H}^{\sharp}\right) \simeq[\rho /(\rho-1)] K_{S^{\sharp}}$. A list of such threefolds (with corresponding values for $\rho$ ) is given in [CF93]. Moreover one can easily calculate $d\left(X^{\sharp}, \mathcal{H}^{\sharp}\right)$ and $n\left(X^{\sharp}, \mathcal{H}^{\sharp}\right)$. Indeed

$$
d\left(X^{\sharp}, \mathcal{H}^{\sharp}\right):=\left(\mathcal{H}^{\sharp}\right)^{3}=\left(\mathcal{O}_{S^{\sharp}}\left(\mathcal{H}^{\sharp}\right)\right)^{2}=\frac{\rho^{2}}{(\rho-1)^{2}} K_{S^{\sharp}}^{2},
$$

and by Riemann-Roch

$$
n\left(X^{\sharp}, \mathcal{H}^{\sharp}\right):=h^{0}\left(\mathcal{H}^{\sharp}\right)-1=h^{0}\left(\mathcal{O}_{S^{\sharp}}\left(\mathcal{H}^{\sharp}\right)\right)=\frac{\rho}{2(\rho-1)^{2}} K_{S^{\sharp}}^{2}+1 .
$$




\section{A. L. Knutsen, C. Novelli And A. Sarti}

TABLE 1. $\mathbb{Q}$-Fano threefolds.

\begin{tabular}{|c|c|c|c|c|c|c|c|}
\hline Type & $X^{\sharp}$ & General $S^{\sharp} \in\left|\mathcal{H}^{\sharp}\right|$ & $\rho$ & $-\frac{\rho}{\rho-1}$ & $K_{S^{\sharp}}^{2}$ & $d\left(X^{\sharp}, \mathcal{H}^{\sharp}\right)$ & $n\left(X^{\sharp}, \mathcal{H}^{\sharp}\right)$ \\
\hline (a) & $\mathbb{P}(1,1,2,3)$ & $H_{6} \subset \mathbb{P}(1,1,2,3)$ & $6 / 7$ & 6 & 1 & 36 & 22 \\
\hline (b) & $T_{6} \subset \mathbb{P}(1,1,2,3,3)$ & $T_{6} \cap\left\{x_{4}=0\right\}$ & $3 / 4$ & 3 & 1 & 9 & 7 \\
\hline (c) & $T_{6} \subset \mathbb{P}(1,1,2,3,4)$ & $T_{6} \cap\left\{x_{4}=0\right\}$ & $4 / 5$ & 4 & 1 & 16 & 11 \\
\hline (d) & $T_{6} \subset \mathbb{P}(1,1,2,3,5)$ & $T_{6} \cap\left\{x_{4}=0\right\}$ & $5 / 6$ & 5 & 1 & 25 & 16 \\
\hline (e) & $T_{6} \subset \mathbb{P}(1,1,2,2,3)$ & $T_{6} \cap\left\{x_{3}=0\right\}$ & $2 / 3$ & 2 & 1 & 4 & 4 \\
\hline (f) & $T_{6} \subset \mathbb{P}(1,1,1,2,3)$ & $T_{6} \cap\left\{x_{0}=0\right\}$ & $1 / 2$ & 1 & 1 & 1 & 2 \\
\hline (g) & $\mathbb{P}(1,1,1,2)$ & $H_{4} \subset \mathbb{P}(1,1,1,2)$ & $4 / 5$ & 4 & 2 & 32 & 21 \\
\hline (h) & $T_{4} \subset \mathbb{P}(1,1,1,2,2)$ & $T_{4} \cap\left\{x_{4}=0\right\}$ & $2 / 3$ & 2 & 2 & 8 & 7 \\
\hline (i) & $T_{4} \subset \mathbb{P}(1,1,1,2,3)$ & $T_{4} \cap\left\{x_{4}=0\right\}$ & $3 / 4$ & 3 & 2 & 18 & 13 \\
\hline$(\mathrm{j})$ & $T_{4} \subset \mathbb{P}(1,1,1,1,2)$ & $T_{4} \cap\left\{x_{0}=0\right\}$ & $1 / 2$ & 1 & 2 & 2 & 3 \\
\hline$(\mathrm{k})$ & $\mathbb{P}^{3}$ & $H_{3} \subset \mathbb{P}^{3}$ & $3 / 4$ & 3 & 3 & 27 & 19 \\
\hline (l) & $T_{3} \subset \mathbb{P}(1,1,1,1,2)$ & $T_{3} \cap\left\{x_{4}=0\right\}$ & $2 / 3$ & 2 & 3 & 12 & 10 \\
\hline (m) & $T_{3} \subset \mathbb{P}^{4}$ & $T_{3} \cap\left\{x_{0}=0\right\}$ & $1 / 2$ & 1 & 3 & 3 & 4 \\
\hline (n) & $T_{2} \subset \mathbb{P}^{4}$ & $H_{2,2} \subset T_{2}$ & $2 / 3$ & 2 & 4 & 16 & 13 \\
\hline (o) & $T_{2,2} \subset \mathbb{P}^{5}$ & $T_{2,2} \cap\left\{x_{0}=0\right\}$ & $1 / 2$ & 1 & 4 & 4 & 5 \\
\hline (p) & $\mathbb{P}^{6} \cap \mathbb{G}(1,4)$ & $\mathbb{P}^{6} \cap \mathbb{G}(1,4) \cap\left\{x_{0}=0\right\}$ & $1 / 2$ & 1 & 5 & 5 & 6 \\
\hline (q) & $T_{2} \subset \mathbb{P}^{4}$ & $T_{2} \cap\left\{x_{0}=0\right\} \simeq \mathbb{P}^{1} \times \mathbb{P}^{1}$ & $1 / 3$ & $1 / 2$ & 8 & 2 & 4 \\
\hline$(\mathrm{r})$ & $\mathbb{P}^{3}$ & $\mathbb{P}^{1} \times \mathbb{P}^{1} \simeq H_{2} \subset \mathbb{P}^{3}$ & $1 / 2$ & 1 & 8 & 8 & 9 \\
\hline$(\mathrm{s})$ & $\mathbb{P}^{3}$ & $\left\{x_{0}=0\right\} \simeq \mathbb{P}^{2} \subset \mathbb{P}^{3}$ & $1 / 4$ & $1 / 3$ & 9 & 1 & 3 \\
\hline$(\mathrm{t})$ & $\mathbb{P}(1,1,1,2)$ & $\left\{x_{3}=0\right\} \simeq \mathbb{P}^{2} \subset \mathbb{P}(1,1,1,2)$ & $2 / 5$ & $2 / 3$ & 9 & 4 & 6 \\
\hline
\end{tabular}

In Table 1 we list all the cases (see [CF93, p. 81]). In the table $\mathbb{P}\left(w_{1}, \ldots, w_{n}\right)$ denotes the weighted projective space with weight $w_{i}$ at the coordinate $x_{i}$. The hyperplane given by $x_{i}$ is denoted $\left\{x_{i}=0\right\}$. Moreover $T_{a}$ (respectively $T_{a, b}$ ) denotes a hypersurface of degree $a$ (respectively a complete intersection of two hypersurfaces of degrees $a$ and $b$ ) and similarly for $H_{a}$ and $H_{a, b}$. The variety $\mathbb{G}(1,4)$ is the Grassmannian parameterizing lines in $\mathbb{P}^{4}$, embedded in $\mathbb{P}^{9}$ by the Plücker embedding.

Definition 1.3. Following [Mel02, Definition 3.3] we will call $\left(X^{\sharp}, \mathcal{H}^{\sharp}\right)$ a $\sharp$-minimal model of the pair $(X, \mathcal{H})$. In particular, by Lemma 1.1 , it exists when $d(X, \mathcal{H})<2 n(X, \mathcal{H})-4$.

Note that a $\sharp$-minimal model exists for any $(X, \mathcal{H})$ with $X$ a terminal $\mathbb{Q}$-factorial uniruled threefold and $\mathcal{H}$ nef with $h^{0}(n \mathcal{H})>1$ for some $n>0$ by [Mel02, Theorem 3.2], but it will in general not have all the nice properties (i) - (v) in Theorem 1.2 above. We will not need the $\sharp$-minimal model in complete generality, but only in the version stated in Theorem 1.2 above.

The following explains the terminology used in Theorem 0.1 and Corollary 0.3.

Definition 1.4. For any pair $(X, \mathcal{H})$ consisting of a threefold $X$ and a big and globally generated line bundle $\mathcal{H}$ on $X$, with $d(X, \mathcal{H})<2 n(X, \mathcal{H})-4$, we will by a $\sharp$-minimal model of $(X, \mathcal{H})$ mean a $\sharp$-minimal model of $\left(\tilde{X}, f^{*} \mathcal{H}\right)$, where $f: \tilde{X} \rightarrow X$ is a minimal resolution of singularities. (Observe that $d\left(\tilde{X}, f^{*} \mathcal{H}\right)=d(X, \mathcal{H})$ and $n\left(\tilde{X}, f^{*} \mathcal{H}\right) \geqslant n(X, \mathcal{H})$, so a $\sharp$-minimal model exists and satisfies the properties (i)-(v) of Theorem 1.2.)

Lemma 1.5. With the same notation and assumptions as in Theorem 1.2, let $S, S^{\sharp}$ and $f$ be as in part (iii) there and set $D:=\mathcal{O}_{S}(\mathcal{H})$.

(a) We have $n\left(X^{\sharp}, \mathcal{H}^{\sharp}\right) \geqslant n(X, \mathcal{H})$ and $d\left(X^{\sharp}, \mathcal{H}^{\sharp}\right) \geqslant d(X, \mathcal{H})$. In particular $\mathcal{H}^{\sharp}$ is big and nef. 


\section{ON VARIETIES THAT ARE UNIRULED BY LINES}

(b) Let $l$ be the total number of irreducible curves contracted by $f$. If $\rho \geqslant 1 / 3$, then

$$
\left(D+\frac{\rho}{1-\rho} K_{S}\right)^{2} \geqslant-l\left(\frac{\rho}{1-\rho}\right)^{2} .
$$

(c) If $\left(X^{\sharp}, \mathcal{H}^{\sharp}\right)$ is of type (I) in Theorem 1.2(v), then

$$
d(X, \mathcal{H})-n(X, \mathcal{H})+1=\frac{\rho(2 \rho-1)}{2(\rho-1)^{2}} K_{S^{\sharp}}^{2}
$$

Proof. We first observe that $n\left(X^{\sharp}, \mathcal{H}^{\sharp}\right) \geqslant n(X, \mathcal{H})$ as $S^{\sharp}=\phi_{*} S$.

We have the commutative diagram

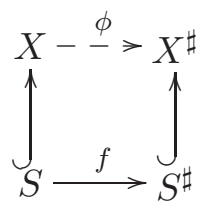

where $f:=\phi_{\mid S}$ is well defined and birational by Theorem 1.2(iii). As observed in [Mel02, Proposition 3.6] one can describe each step in the $\sharp$-minimal model program in a neighborhood of $S$. More precisely, set $X_{0}:=X, S_{0}:=S, X_{m}:=X^{\sharp}$ and $S_{m}=S^{\sharp}:=\phi_{*} S$. Denote by $\phi_{i}: X_{i-1}->X_{i}$ for $i=1, \ldots, m$ each birational modification in the $\sharp$-minimal model program relative to $(X, \mathcal{H})$ and define inductively $S_{i}:=\phi_{*} S_{i-1}$. Then each $S_{i}$ is smooth, and setting $f_{i}:=\phi_{i \mid S_{i}}$ we can factorize $f$ as

$$
S \stackrel{f_{1}}{\longrightarrow} S_{1} \stackrel{f_{2}}{\longrightarrow} \cdots \stackrel{f_{m-1}}{\longrightarrow} S_{m-1} \stackrel{f_{m}}{\longrightarrow} S_{m}=S^{\sharp},
$$

where each $f_{i}$ contracts $l_{i}$ disjoint $(-1)$-curves $E_{1}^{i}, \ldots, E_{l_{i}}^{i}$ with $l_{i} \geqslant 0$ by [Mel02, Proposition 3.6]. The total number of contracted curves is $l=\sum_{i=1}^{m} l_{i}$. We set $D_{i}:=\mathcal{O}_{S_{i}}\left(S_{i}\right)$ and $D^{\sharp}:=\mathcal{O}_{S^{\sharp}}\left(S^{\sharp}\right)$.

If $\phi_{i}$ is a flip then $S_{i}$ is disjoint from the flipping curves by [Mel02, Claim 3.7], so that $f_{i}$ is an isomorphism.

If $\phi_{i}$ contracts a divisor onto a curve then it is shown in [Mel02, Case 3.8] that the fiber $F_{i}$ of $\phi_{i}$ satisfies $S_{i} \cdot F_{i}=0$, whence $D_{i} \cdot F_{i}=0$, which means that all $E_{j}^{i}$ satisfy $E_{j}^{i} \cdot D_{i}=0$.

If $\phi_{i}$ contracts a divisor onto a point then it is shown in [Mel02, Case 3.9] that $f_{i}$ is a contraction of a single (-1)-curve $E_{i}=E_{1}^{i}$ that satisfies $E_{i} \cdot D_{i}=1$.

In other words, for every $i$ we have three possibilities:

$$
\begin{aligned}
& l_{i}=0 ; \quad \text { or } \\
& l_{i}>0 \text { and } E_{j}^{i} \cdot D_{i}=0 \text { for all } j \in\left\{1, \ldots, l_{i}\right\} ; \text { or } \\
& l_{i}=1 \text { and } E_{1}^{i} \cdot D_{i}=1 .
\end{aligned}
$$

Now denote by $L_{j}^{i}$ the total transform of $E_{j}^{i}$ on $S$. Then $\left(L_{j}^{i}\right)^{2}=-1$ and $L_{j}^{i} \cdot L_{j^{\prime}}^{i^{\prime}}=0$ for $(i, j) \neq\left(i^{\prime}, j^{\prime}\right)$. We have

and, by (12),

$$
K_{S}=f^{*} K_{S^{\sharp}}+\sum L_{j}^{i}
$$

In particular

$$
D=f^{*} D^{\sharp}-\sum \mu_{j}^{i} L_{j}^{i} \quad \text { with } \mu_{j}^{i} \in\{0,1\} .
$$

$$
d(X, \mathcal{H})=D^{2}=\left(D^{\sharp}\right)^{2}-\sum\left(\mu_{j}^{i}\right)^{2}=d\left(X^{\sharp}, \mathcal{H}^{\sharp}\right)-\sum \mu_{j}^{i} \leqslant d\left(X^{\sharp}, \mathcal{H}^{\sharp}\right),
$$

finishing the proof of part (a). 


\section{A. L. Knutsen, C. Novelli And A. Sarti}

From (13) and (14) we get

$$
D+\frac{\rho}{1-\rho} K_{S}=f^{*}\left(D^{\sharp}+\frac{\rho}{1-\rho} K_{S^{\sharp}}\right)+\sum\left(\frac{\rho}{1-\rho}-\mu_{j}^{i}\right) L_{j}^{i},
$$

and since there are $l$ terms in the sum we get

$$
\left(D+\frac{\rho}{1-\rho} K_{S}\right)^{2}=\left(D^{\sharp}+\frac{\rho}{1-\rho} K_{S^{\sharp}}\right)^{2}-l\left(\frac{\rho}{1-\rho}\right)^{2}+\sum \mu_{j}^{i}\left(\frac{2 \rho}{1-\rho}-\mu_{j}^{i}\right) .
$$

By definition and invariance of $\rho$ (cf. Theorem 1.2(i)) we have that $\rho K_{T^{\sharp}}+\mathcal{H}^{\sharp}$ is $\mathbb{Q}$-effective. From Theorem 1.2(ii) we have that it is also $\mathbb{Q}$-nef, whence its restriction to $S^{\sharp}$ is also $\mathbb{Q}$-effective and $\mathbb{Q}$-nef. Since $S^{\sharp}$ is Cartier we get by adjunction that

$$
\left(\rho K_{T^{\sharp}}+\mathcal{H}^{\sharp}\right)_{\mid S^{\sharp}} \simeq(1-\rho)\left(\frac{\rho}{1-\rho} K_{S^{\sharp}}+D^{\sharp}\right),
$$

whence by $\mathbb{Q}$-nefness

$$
\left(D^{\sharp}+\frac{\rho}{1-\rho} K_{S \sharp}\right)^{2} \geqslant 0 .
$$

Moreover the assumption $\rho \geqslant \frac{1}{3}$ is equivalent to $\rho /(1-\rho) \geqslant \frac{1}{2}$, whence

$$
\sum \mu_{j}^{i}\left(\frac{2 \rho}{1-\rho}-\mu_{j}^{i}\right) \geqslant \mu_{j}^{i}\left(1-\mu_{j}^{i}\right) \geqslant 0
$$

Now (10) in part (b) follows combining (16)-(18).

We have left to prove part (c). Since $S^{\sharp}$ is a smooth del Pezzo surface, we have $h^{1}\left(\mathcal{O}_{S}\right)=$ $h^{1}\left(\mathcal{O}_{S^{\sharp}}\right)=0$. It is then easily seen by the proof of Lemma 1.1 that equality holds in (3) (note that we have $h^{1}\left(\mathcal{O}_{X}\right) \leqslant h^{1}\left(\mathcal{O}_{X}(-\mathcal{H})\right)+h^{1}\left(\mathcal{O}_{S}\right)=0$ by Kawamata-Viehweg vanishing). Using (13), (14) and (15) we therefore get, for $D \in\left|\mathcal{O}_{S}(\mathcal{H})\right|$,

$$
\begin{aligned}
d(X, \mathcal{H})-2 n(X, \mathcal{H})+2 & =D \cdot K_{S} \\
& =\left(f^{*} D^{\sharp}-\sum \mu_{j}^{i} L_{j}^{i}\right) \cdot\left(f^{*} K_{S^{\sharp}}+\sum L_{j}^{i}\right) \\
& =D^{\sharp} \cdot K_{S^{\sharp}}+\sum \mu_{j}^{i} \\
& =\frac{\rho}{\rho-1} K_{S^{\sharp}}^{2}+\sum \mu_{j}^{i} \\
& =\frac{\rho}{\rho-1} K_{S^{\sharp}}^{2}+d\left(X^{\sharp}, \mathcal{H}^{\sharp}\right)-d(X, \mathcal{H}) .
\end{aligned}
$$

Now using (8) we obtain

$$
2 d(X, \mathcal{H})-2 n(X, \mathcal{H})+2=\frac{\rho(2 \rho-1)}{(\rho-1)^{2}} K_{S^{\sharp}}^{2}
$$

proving part (c).

\section{Bounds for uniruledness degree one}

As a 'warming up' before proceeding with the proofs of the main results we give the proof of the following bound.

Proposition 2.1. Let $(X, \mathcal{H})$ be a pair consisting of a terminal $\mathbb{Q}$-factorial threefold $X$ and a globally generated and big line bundle $\mathcal{H}$ on $X$. Set $d:=d(X, \mathcal{H})$ and $n:=n(X, \mathcal{H})$.

If $n \geqslant 4, d<\frac{4}{3} n-\frac{4}{3}$ and $d \neq n-1$ for $n \leqslant 9$, then $X$ is uniruled of $\mathcal{H}$-degree one. 


\section{ON VARIETIES THAT ARE UNIRULED BY LINES}

Proof. Since $n \geqslant 4$ we have $2 n-4 \geqslant \frac{4}{3} n-\frac{4}{3}$, whence $d<2 n-4$, so by Lemma 1.1(i) the general $S \in|\mathcal{H}|$ is smooth of negative Kodaira dimension.

Moreover for any irreducible $D \in\left|\mathcal{H}_{\mid S}\right|$ we have, by Lemma 1.1(ii),

$$
\begin{aligned}
D \cdot\left(\frac{3}{2} \mathcal{H}+K_{X}\right) & =D \cdot\left(\mathcal{H}+K_{X}\right)+\frac{1}{2} D \cdot \mathcal{H}=D \cdot K_{S}+\frac{1}{2} d \\
& \leqslant d-2 n+2+\frac{1}{2} d=\frac{3}{2} d-2 n+2 \\
& <\frac{3}{2}\left(\frac{4}{3} n-\frac{4}{3}\right)-2 n+2=0,
\end{aligned}
$$

whence $\rho(X, \mathcal{H})<2 / 3$.

It follows that the $\sharp$-minimal model $\left(X^{\sharp}, \mathcal{H}^{\sharp}\right)$ is in the list of Theorem $1.2(\mathrm{v})$ and moreover it cannot be as in (II) since $\rho(X, \mathcal{H})=2 / 3$ in this case. In the cases (III)-(V) one immediately sees that $\left(X^{\sharp}, \mathcal{H}^{\sharp}\right)$ is uniruled of $\mathcal{H}^{\sharp}$-degree one, whence $(X, \mathcal{H})$ is also uniruled of $\mathcal{H}$-degree one by Theorem 1.2(iv).

We have $n\left(T^{\sharp}, \mathcal{H}^{\sharp}\right) \geqslant n \geqslant 4$ by Lemma $1.5(\mathrm{a})$, and by using Table 1 we see that the cases in (I) where $n\left(X^{\sharp}, \mathcal{H}^{\sharp}\right) \geqslant 4$ and $\rho<2 / 3$ are the cases (m), (o), (p), (q), (r) and (t). Among these all but (r) are clearly uniruled of $\mathcal{H}^{\sharp}$-degree one.

By (11) and Table 1 we have $d-n+1=0$ in case $(r)$ and by Lemma 1.5 (a) we have $n \leqslant$ $n\left(X^{\sharp}, \mathcal{H}^{\sharp}\right)=9$.

Corollary 2.2. Let $(X, \mathcal{H})$ be a pair consisting of a threefold $X$ and a globally generated and big line bundle $\mathcal{H}$ on $X$. Set $d:=d(X, \mathcal{H})$ and $n:=n(X, \mathcal{H})$.

If $d<\frac{4}{3} n-\frac{4}{3}, d \neq n$ when $5 \leqslant n \leqslant 8$ and $d \neq n-1$ for $n \leqslant 9$, then $X$ is uniruled of $\mathcal{H}$-degree one.

Proof. Let $\pi: \tilde{X} \rightarrow X$ be a resolution of the singularities of $X$. Then $\pi^{*} \mathcal{H}$ is globally generated and big with $d\left(\tilde{X}, \pi^{*} \mathcal{H}\right)=\mathcal{H}^{3}=d$ and $n\left(\tilde{X}, \pi^{*} \mathcal{H}\right)=\operatorname{dim}\left|\pi^{*} \mathcal{H}\right| \geqslant n$ and we can apply Proposition 2.1. The additional cases $d=n$ for $5 \leqslant n \leqslant 8$ occur since equality does not need to occur in $n\left(\tilde{X}, \pi^{*} \mathcal{H}\right) \geqslant n$.

Remark 2.3. We note that the last corollary improves Theorem 0.1 for $n \leqslant 12$. Moreover, the cases $n=3,4$ are trivial, as are the cases $n=5,6,7$, since then $\varphi_{\mathcal{H}}(X) \subseteq \mathbb{P}^{n}$ has minimal degree. Hence the relevant statement, combining Theorem 0.1 and Corollary 2.2 is: $X$ is uniruled of $\mathcal{H}$-degree one in the following cases:

(i) $n=8$ and $d=6$ or 9 ;

(ii) $n=9$ and $d=7,9$ or 10 ;

(iii) $n=10$ and $d \leqslant 11$;

(iv) $n=11$ or 12 and $d \leqslant n+2$;

(v) $n \geqslant 13$ and $d \leqslant 2 n-11,(d, n) \neq(27,19)$.

Now we give the main ideas and the strategy of the proof of Theorem 0.1 . The main result we will need to prove is the following.

Proposition 2.4. Let $(X, \mathcal{H})$ be a pair consisting of a terminal $\mathbb{Q}$-factorial threefold $X$ and a globally generated, big line bundle $\mathcal{H}$ on $X$. Set $d:=d(X, \mathcal{H})$ and $n:=n(X, \mathcal{H})$.

If a $\sharp$-minimal model of $(X, \mathcal{H})$ is of type (II) in Theorem $1.2(\mathrm{v})$, then $d \geqslant 2 n-10$.

The proof of this result will be given in $\S 3$ below, after a careful study of the threefolds of type (II) in Theorem 1.2(v). We will now give the proofs of Theorem 0.1 and Corollaries 0.2 and 0.3 assuming Proposition 2.4. 


\section{A. L. Knutsen, C. Novelli and A. Sarti}

Proof of Theorem 0.1. Let $X$ be a reduced and irreducible three-dimensional variety and $\mathcal{H}$ a globally generated big line bundle on $X$. Set $d:=\mathcal{H}^{3}$ and $n:=h^{0}(X, \mathcal{H})-1$ and assume $d<2 n-10$.

Let $\pi: \tilde{X} \rightarrow X$ be a resolution of the singularities of $X$. Then $\pi^{*} \mathcal{H}$ is globally generated and big with $d\left(\tilde{X}, \pi^{*} \mathcal{H}\right)=\mathcal{H}^{3}=d$ and $n\left(\tilde{X}, \pi^{*} \mathcal{H}\right)=\operatorname{dim}\left|\pi^{*} \mathcal{H}\right| \geqslant n$. Since $(d, n)=(27,19)$ satisfies $d=2 n-11$ we can reduce to the case where $X$ is smooth. Therefore we assume $X$ is smooth.

By Lemma 1.1(i), any $\sharp$-minimal model $\left(X^{\sharp}, \mathcal{H}^{\sharp}\right)$ of $(X, \mathcal{H})$ is in the list of Theorem $1.2(\mathrm{v})$. Moreover, by Proposition 2.4, it cannot be of type (II).

We easily see that the cases (III)-(V) are uniruled of $\mathcal{H}^{\sharp}$-degree one. In the cases (I) we have, by Lemma 1.5,

$$
\frac{\rho(2 \rho-1)}{2(\rho-1)^{2}} K_{S^{\sharp}}^{2}=d-n+1 \leqslant n-10 \leqslant n\left(X^{\sharp}, \mathcal{H}^{\sharp}\right)-10 .
$$

By checking Table 1 one finds that we can only be in case $(\mathrm{k})$, with equalities all the way in (19). Hence $n=n\left(X^{\sharp}, \mathcal{H}^{\sharp}\right)=19$ and $d=2 n-11=27$. Now the result follows from Theorem 1.2(iv).

Proof of Corollary 0.2. Let $X$ be a reduced and irreducible variety of dimension $k \geqslant 4$ and $\mathcal{H}$ a globally generated big line bundle on $X$ with $d:=\mathcal{H}^{k}$ and $n:=h^{0}(X, \mathcal{H})-1$.

As just mentioned in the proof of Theorem 0.1 we can assume that $X$ is smooth.

Setting $X_{k}:=X$ and $\mathcal{H}_{k}:=\mathcal{H}$, we recursively choose general smooth 'hyperplane sections' $X_{i-1} \in\left|\mathcal{H}_{i}\right|$ and define $\mathcal{H}_{i-1}:=\mathcal{H}_{i} \otimes \mathcal{O}_{X_{i-1}}$, for $2 \leqslant i \leqslant k$. (Note that $\operatorname{dim} X_{i}=i$ and $\mathcal{H}_{i}$ is a line bundle on $X_{i}$.)

Let $n_{3}:=h^{0}\left(\mathcal{H}_{3}\right)-1$. Then from the exact sequence

$$
0 \longrightarrow \mathcal{O}_{X_{i}} \longrightarrow \mathcal{H}_{i} \longrightarrow \mathcal{H}_{i-1} \longrightarrow 0
$$

we have

$$
n_{3} \geqslant n-(k-3)=n-k+3 .
$$

Together with the condition $d<2(n-k)-4$ this implies $d<2 n_{3}-10$ and it follows from Theorem 0.1 that either $\left(X_{3}, \mathcal{H}_{3}\right)$ is uniruled of degree one or $\left(d, n_{3}\right)=(27,19)$ and $\left(X_{3}^{\sharp}, \mathcal{H}_{3}^{\sharp}\right)$ is $\left(\mathbb{P}^{3}, \mathcal{O}(3)\right)$.

In the second case we have equality in $(21)$, i.e.

$$
19=n_{3}=h^{0}\left(\mathcal{H}_{3}\right)-1=h^{0}(\mathcal{H})-(k-3)-1=n-k+3 .
$$

Denote by $\phi: X_{3}-->X_{3}^{\sharp}=\mathbb{P}^{3}$ the birational map of the $\sharp$-minimal model program. By Theorem 1.2(iii) its restriction $f$ to $S:=X_{2}$ is a birational morphism onto a smooth surface $S^{\sharp} \in\left|\mathcal{O}_{\mathbb{P}^{3}}(3)\right|$.

We have

$$
19=h^{0}\left(\mathcal{O}_{S^{\sharp}}(3)\right) \geqslant h^{0}\left(\mathcal{H}_{2}\right) \geqslant h^{0}\left(\mathcal{H}_{3}\right)-1=19
$$

by Lemma $1.5(\mathrm{a})$ and $(22)$, whence $\left|\mathcal{O}_{S^{\sharp}}(3)\right|=f_{*}\left|\mathcal{H}_{2}\right|$ and this can only be base point free if every curve $E$ contracted by $f$ satisfies $E \cdot \mathcal{H}_{2}=0$. Denoting by $\varphi_{\mathcal{H}_{2}}$ and $\varphi_{\mathcal{O}_{S}(3)}$ the morphisms defined by $\left|\mathcal{H}_{2}\right|$ and $\left|\mathcal{O}_{S^{\sharp}}(3)\right|$ respectively, this implies that $\varphi_{\mathcal{O}_{S \sharp}(3)} \circ f=\varphi_{\mathcal{H}_{2}}$; in other words $S^{\prime}:=\varphi_{\mathcal{H}_{2}}(S)=\varphi_{\mathcal{O}_{S^{\sharp}}(3)}\left(S^{\sharp}\right) \simeq S^{\sharp} \subseteq \mathbb{P}^{18}$. Moreover, by $(22)$, the natural map $H^{0}(\mathcal{H}) \rightarrow H^{0}\left(\mathcal{H}_{2}\right)$ is surjective, so $S^{\prime}=\varphi_{\mathcal{H}}(S)$, where $\varphi_{\mathcal{H}}: X \rightarrow \mathbb{P}^{n}$ is the morphism defined by $|\mathcal{H}|$. Note that $\varphi_{\mathcal{H}}$ is birational for reasons of degree. Setting $X^{\prime}:=\varphi_{\mathcal{H}}(X) \subseteq \mathbb{P}^{n}$ we therefore have that $S^{\prime} \subseteq X^{\prime}$ is a smooth, linear, transversal surface section (recall that $S \subseteq X$ is a complete intersection of $(k-2)$ general elements of $|\mathcal{H}|)$.

We now apply the theorem of Zak (unpublished, cf. [Zak91]) and L'vovsky (cf. [Lvo92a] and [Lvo92b]), which says the following (cf. [Lvo92b, Theorem 0.1]). If $V \subsetneq \mathbb{P}^{N}$ is a smooth, nondegenerate variety that is not a quadric and satisfies $h^{0}\left(\mathcal{N}_{V / \mathbb{P}^{N}}(-1)\right)<2 N+1$, if $Y \subseteq \mathbb{P}^{N+m}$ is a nondegenerate, irreducible $(m+\operatorname{dim} V)$-dimensional variety with $m>h^{0}\left(\mathcal{N}_{V / \mathbb{P}^{N}}(-1)\right)-N-1$, and 


\section{ON VARIETIES THAT ARE UNIRULED BY LINES}

if $L=\mathbb{P}^{N} \subseteq \mathbb{P}^{N+m}$ is a linear subspace such that $V=L \cap Y$ (scheme-theoretically), then $Y$ is a cone.

Since a cone is uniruled by lines, the corollary will follow if we show that $h^{0}\left(\mathcal{N}_{S^{\prime} / \mathbb{P}^{18}}(-1)\right) \leqslant 20$, with $S^{\prime}$ being the 3 -ple embedding of a smooth cubic surface $S_{0}$ in $\mathbb{P}^{3}$.

We argue as in [GLM04, pp. 160-161] to compute $h^{0}\left(\mathcal{N}_{S^{\prime} / \mathbb{P}^{18}}(-1)\right)$. We give the argument for the sake of the reader.

From the Euler sequence and tangent bundle sequence

$$
\begin{gathered}
0 \longrightarrow \mathcal{O}_{S^{\prime}}(-1) \longrightarrow \mathbb{C}^{19} \otimes \mathcal{O}_{S^{\prime}} \longrightarrow \mathcal{T}_{\mathbb{P}^{18}}(-1) \otimes \mathcal{O}_{S^{\prime}} \longrightarrow 0 \\
0 \longrightarrow \mathcal{T}_{S^{\prime}}(-1) \longrightarrow \mathcal{T}_{\mathbb{P}^{18}}(-1) \otimes \mathcal{O}_{S^{\prime}} \longrightarrow \mathcal{N}_{S^{\prime} / \mathbb{P}^{18}}(-1) \longrightarrow 0,
\end{gathered}
$$

we find

$$
h^{0}\left(\mathcal{N}_{S^{\prime} / \mathbb{P}^{18}}(-1)\right) \leqslant h^{0}\left(\mathcal{T}_{\mathbb{P} 18}(-1) \otimes \mathcal{O}_{S^{\prime}}\right)+h^{1}\left(\mathcal{T}_{S^{\prime}}(-1)\right)=19+h^{1}\left(\mathcal{T}_{S_{0}}(-3)\right) .
$$

From the tangent bundle sequence of $S_{0} \subseteq \mathbb{P}^{3}$,

$$
0 \longrightarrow \mathcal{T}_{S_{0}}(-3) \longrightarrow \mathcal{T}_{\mathbb{P} 3}(-3) \otimes \mathcal{O}_{S_{0}} \longrightarrow \mathcal{N}_{S_{0} / \mathbb{P}^{3}}(-3) \longrightarrow 0,
$$

and the fact that $\mathcal{N}_{S_{0} / \mathbb{P}^{3}}(-3) \simeq \mathcal{O}_{S_{0}}$, we find

$$
h^{1}\left(\mathcal{T}_{S_{0}}(-3)\right) \leqslant 1+h^{1}\left(\mathcal{T}_{\mathbb{P}^{3}}(-3) \otimes \mathcal{O}_{S_{0}}\right) .
$$

In view of $(23)$ it will suffice to show that $h^{1}\left(\mathcal{T}_{\mathbb{P} 3}(-3) \otimes \mathcal{O}_{S_{0}}\right)=0$.

Now observe that $\mathcal{T}_{\mathbb{P}^{3}}(-3) \simeq\left(\Omega_{\mathbb{P}^{3}}^{1}\right)^{\vee} \otimes K_{\mathbb{P}^{3}} \otimes \mathcal{O}_{\mathbb{P}^{3}}(1) \simeq \Omega_{\mathbb{P}^{3}}^{2}(1)$ so using Bott vanishing on $\mathbb{P}^{3}$ and Serre duality one gets $h^{1}\left(\mathcal{T}_{\mathbb{P}^{3}}(-3)\right)=0$ and

$$
h^{2}\left(\mathcal{T}_{\mathbb{P}^{3}}(-6)\right)=h^{2}\left(\left(\Omega_{\mathbb{P}^{3}}^{1}\right)^{\vee} \otimes K_{\mathbb{P}^{3}} \otimes \mathcal{O}_{\mathbb{P}^{3}}(-2)\right)=h^{1}\left(\Omega_{\mathbb{P}^{3}}^{2}(2)\right)=0 .
$$

This yields $h^{1}\left(\mathcal{T}_{\mathbb{P}^{3}}(-3) \otimes \mathcal{O}_{S_{0}}\right)=0$.

This concludes the proof of Corollary 0.2.

It is immediate that Corollary 0.3 follows from Theorem 0.1 and Corollary 0.2.

As we already noted in the Introduction, Theorem 0.1 is sharp by the following example.

Example 2.5. The bound of Theorem 0.1 is sharp. In fact consider $X=\mathbb{P}^{2} \times \mathbb{P}^{1}$ with projections $p$ and $q$ respectively and let $\mathcal{H}:=p^{*} \mathcal{O}_{\mathbb{P}^{2}}(2) \otimes q^{*} \mathcal{O}_{\mathbb{P}^{1}}(a)$ for an integer $a>0$. We have

$$
n:=h^{0}(\mathcal{H})-1=h^{0}\left(\mathcal{O}_{\mathbb{P}^{2}}(2)\right) \cdot h^{0}\left(\mathcal{O}_{\mathbb{P}^{1}}(a)\right)-1=6(a+1)-1
$$

and

$$
d:=\mathcal{H}^{3}=\left(p^{*} \mathcal{O}(2) \otimes q^{*} \mathcal{O}(a)\right)^{3}=3\left(p^{*} \mathcal{O}(2)\right)^{2} \cdot q^{*} \mathcal{O}(a)=12 a,
$$

whence $d=2 n-10$.

If $a \geqslant 2$, then clearly any curve $C$ on $X$ satisfies

$$
C \cdot \mathcal{H}=C \cdot p^{*} \mathcal{O}_{\mathbb{P}^{2}}(2)+C \cdot q^{*} \mathcal{O}_{\mathbb{P}^{1}}(a) \geqslant 2,
$$

with equality obtained for the lines in the $\mathbb{P}^{2}$-fibers, so that $X$ is uniruled of $\mathcal{H}$-degree two and not uniruled of $\mathcal{H}$-degree one.

If $a=1$, then $X$ is clearly uniruled of $\mathcal{H}$-degree one, and since $d=12$ and $n=11$, this also follows from Remark 2.3.

\section{Terminal Veronese fibrations}

In this section we will prove Propositions 0.4 and 2.4 . 


\section{A. L. Knutsen, C. Novelli And A. Sarti}

Since we will have to study the threefolds as in item (II) of Theorem 1.2(v) we find it convenient to make the following definition.

Definition 3.1. Let $(T, \mathcal{L})$ be a pair satisfying the following.

(i) $T$ is a terminal $\mathbb{Q}$-factorial threefold with a Mori fiber space structure $p: T \rightarrow B$, where $B$ is a smooth curve.

(ii) $\mathcal{L}$ is a line bundle on $T$ such that the system $|\mathcal{L}|$ contains a smooth surface and has at most base points and $\mathcal{L}^{3}>0$.

(iii) The general fiber of $p$ is $\left(V, \mathcal{L}_{\mid V}\right) \simeq\left(\mathbb{P}^{2}, \mathcal{O}_{\mathbb{P}^{2}}(2)\right)$ and the rest are at most finitely many fibers $\left(G, \mathcal{L}_{\mid G}\right) \simeq\left(\mathbf{S}_{4}, \mathcal{O}_{\mathbf{S}_{4}}(1)\right)$, where $\mathbf{S}_{4} \subset \mathbb{P}^{5}$ is the cone over a normal quartic curve.

Such a Mori fiber space will be called a (three-dimensional) terminal Veronese fibration.

The threefolds of type (II) in Theorem 1.2(v) are terminal Veronese fibrations.

The easiest examples of terminal Veronese fibrations are the smooth ones in Example 2.5. However, there are also singular such varieties and these were erroneously left out in both [Mel97, Proposition 3.7] and [CF93, Proposition 3.4], as remarked by Mella in [Mel02, Remark 5.4]: Take $\mathbb{P}^{2} \times \mathbb{P}^{1}$ and blow up a conic $C$ in a fiber and contract the strict transform of $C$, thus producing a Veronese cone singularity.

Although our main aim is to prove Proposition 2.4 we believe that terminal Veronese fibrations are interesting in their own right. In order to prove Proposition 2.4 we will study 'hyperplane sections' of $T$, i.e. surfaces in $|\mathcal{L}|$, and show that the desired bound on the degree follows since the general such surface has to have a certain number of degenerate fibers, i.e. unions of two conics (with respect to $\mathcal{L}$ ). What we first prove in this section is the following, which is part of the statement in Proposition 0.4.

Proposition 3.2. Let $(T, \mathcal{L})$ be a three-dimensional terminal Veronese fibration and set $n:=$ $h^{0}(\mathcal{L})-1$ and $d:=\mathcal{L}^{3}$.

Then any smooth member of $|\mathcal{L}|$ is a surface fibered over $B$ with $k \geqslant \frac{1}{2}(n-5)$ fibers that are unions of two smooth rational curves intersecting in one point (the other fibers are smooth rational curves).

Proof. Denote by $\mathcal{V}$ the numerical equivalence class of a fiber. Let $S \in|\mathcal{L}|$ be a smooth surface. Then, since $T$ is terminal, we have $S \cap \operatorname{Sing} T=\emptyset$ (cf. [Mel02, (2.3)]).

By property (iii) of Definition 3.1 any fiber of $S$ over $B$ is either a smooth quartic, a union of two conics intersecting in one point, or a double conic, all with respect to $\mathcal{L}$. Denote by $F$ the numerical equivalence class in $S$ of a fiber over $B$. Then $F^{2}=0$.

If a fiber were a double conic, we could write $F \equiv 2 F_{0}$ in Num $S$. However, in this case we would get the contradiction $F_{0} \cdot\left(F_{0}+K_{S}\right)=-1$, so this case does not occur.

In the case of a fiber that is a union of two conics intersecting in one point, we have $F \equiv F_{1}+F_{2}$ in Num $S$, whence by adjunction both $F_{i}$ are $(-1)$-curves. Since $S$ is smooth its general fiber over $B$ is a smooth quartic (with $F \cdot K_{S}=-2$ by adjunction), whence $S$ has a finite number $k$ of degenerate fibers that are unions of two conics, and since these are all $(-1)$-curves we can blow down one of these curves in every fiber and reach a minimal model $R$ for $S$ which is a ruled surface over $B$. Let $g$ be the genus of $B$. Then

$$
k=K_{R}^{2}-K_{S}^{2}=8(1-g)-K_{S}^{2}=8(1-g)-\left(K_{T}+\mathcal{L}\right)^{2} \cdot \mathcal{L},
$$

which only depends on the numerical equivalence class of $S$. Therefore, any smooth surface in $|\mathcal{L}|$ has the same number of degenerate fibers. 


\section{ON VARIETIES THAT ARE UNIRULED BY LINES}

Now note that the fibers of $S$ over the finitely many points of $B$ over which $T$ has singular fibers are all smooth quartics, since $S \cap \operatorname{Sing} T=\emptyset$.

We now consider the birational map of $T$ to a smooth projective bundle $\tilde{T}$, as described in [Mel02, p. 699].

Around one singular fiber $\mathbf{S}_{4}$ of $T$ over a point $p \in B$ this map is given by a succession of blow-ups $\nu_{i}$ and contractions $\mu_{i}$ :

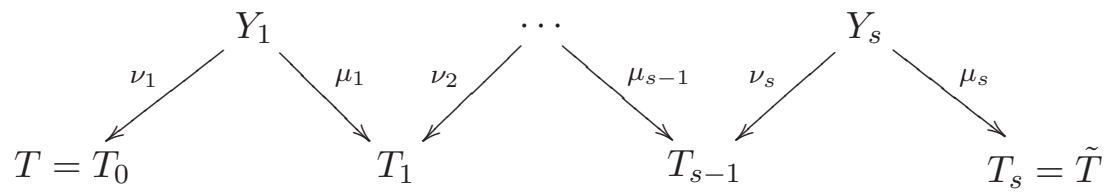

where the procedure ends as soon as some $T_{s}$ has a fiber over $p \in B$ that is a smooth Veronese surface.

For every $\nu_{i}$ the exceptional divisor $E_{i}$ is either a smooth Veronese surface or a cone over a rational normal quartic curve, and the strict transform of the singular fiber $\mathbf{S}_{4}$ of $T_{i-1}$ over $p$ is $G_{i} \simeq \mathbb{F}_{4}$, the desingularization of $\mathbf{S}_{4}$. These two intersect along a smooth quartic $C_{i}$. Then $\mu_{i}$ contracts $G_{i}$ onto a smooth quartic curve $C_{i}^{\prime}=\mu_{i}\left(C_{i}\right)$ and $T_{i}$ is smooth along the exceptional locus of the contraction.

Following $S$ throughout the procedure, we see that $S$ stays out of the exceptional locus of every $\nu_{i}$ and in the contraction it is mapped to a surface having $C_{i}^{\prime}$ as fiber over $p$.

In other words the procedure of desingularizing one singular fiber of $T$ maps every smooth surface in $|\mathcal{L}|$ to a smooth surface passing through a unique smooth quartic over $p$.

Doing the same procedure for all the other singular fibers of $T$ we therefore reach a smooth projective bundle $\mathbb{P}(\mathcal{E})$ over $B$ and under this process $|\mathcal{L}|$ is 'mapped' to a (not necessarily complete) linear system on $\mathbb{P}(\mathcal{E})$ having smooth quartics over the corresponding points of $B$ as base curves. Denote the corresponding line bundle on $\mathbb{P}(\mathcal{E})$ by $\mathcal{L}^{\prime}$. Since we have not changed the number of degenerate fibers of any smooth surface in $|\mathcal{L}|$ over $B$, we see that every smooth surface in $\left|\mathcal{L}^{\prime}\right|$ still has $k$ degenerate fibers over $B$. Since clearly $\operatorname{dim}\left|\mathcal{L}^{\prime}\right| \geqslant \operatorname{dim}|\mathcal{L}|$ it is now sufficient to show that any smooth surface in $\left|\mathcal{L}^{\prime}\right|$ has $k \geqslant \frac{1}{2}\left(h^{0}\left(\mathcal{L}^{\prime}\right)-6\right)$ fibers that are unions of two conics (with respect to $\mathcal{L}^{\prime}$ ) intersecting in one point. This is the content of the following proposition.

Proposition 3.3. Let $f: T \simeq \mathbb{P}(\mathcal{E}) \rightarrow B$ be a three-dimensional projective bundle over a smooth curve of genus $g$. Assume $\mathcal{L}$ is a line bundle on $T$ satisfying:

(i) $\mathcal{L}_{\mid V} \simeq \mathcal{O}_{\mathbb{P}^{2}}(2)$ for every fiber $V \simeq \mathbb{P}^{2}$;

(ii) $|\mathcal{L}|$ is nonempty with general element a smooth irreducible surface;

(iii) the only curves in the base locus of $|\mathcal{L}|$, if any, are smooth quartics (with respect to $\mathcal{L}$ ) in the fibers.

Then any smooth surface in $|\mathcal{L}|$ is fibered over $B$ with $k$ fibers that are unions of two conics (with respect to $\mathcal{L}$ ) intersecting in one point, where

$$
k=\frac{1}{4} \mathcal{L}^{3} \geqslant \frac{1}{2}\left(h^{0}(\mathcal{L})-6\right) .
$$

Proof. We only have to prove (26).

Denote by $\mathcal{V}$ the numerical equivalence class of a fiber. Since every fiber of $T$ over $B$ is a $\mathbb{P}^{2}$ we have $\left(K_{T}\right)_{\mathbb{P}^{2}} \simeq K_{\mathbb{P}^{2}} \simeq \mathcal{O}_{\mathbb{P}^{2}}(-3)$ so we can choose a very ample line bundle $\mathcal{G} \in \operatorname{Pic} T$ such that

$$
\begin{gathered}
\operatorname{Num} T \simeq \mathbb{Z} \mathcal{G} \oplus \mathbb{Z} \mathcal{V}, \quad \mathcal{G}^{2} \cdot \mathcal{V}=1, \quad \mathcal{G} \cdot \mathcal{V}^{2}=\mathcal{V}^{3}=0 \\
K_{T} \equiv b \mathcal{V}-3 \mathcal{G}, \quad b \in \mathbb{Z} \\
\mathcal{L} \equiv a \mathcal{V}+2 \mathcal{G}, \quad a \in \mathbb{Z}
\end{gathered}
$$




\section{A. L. Knutsen, C. Novelli And A. Sarti}

The general element $G \in|\mathcal{G}|$ is a smooth ruled surface over $B$; in particular

$$
8(1-g)=K_{G}^{2}=\left(K_{T}+\mathcal{G}\right)^{2} \cdot \mathcal{G}=(b \mathcal{V}-2 \mathcal{G})^{2} \cdot \mathcal{G}=4 \mathcal{G}^{3}-4 b,
$$

that is

$$
\mathcal{G}^{3}=2(1-g)+b
$$

Let now $S \in|\mathcal{L}|$ be any smooth surface. Clearly (as discussed in the proof of the previous proposition) $K_{S}^{2}=8(1-g)-k$. We compute, using (27) and (30),

$$
\begin{aligned}
K_{S}^{2} & =\left(K_{T}+\mathcal{L}\right)^{2} \cdot \mathcal{L}=((a+b) \mathcal{V}-\mathcal{G})^{2} \cdot(a \mathcal{V}+2 \mathcal{G}) \\
& =2 \mathcal{G}^{3}-3 a-4 b=2(2(1-g)+b)-3 a-4 b \\
& =4(1-g)-3 a-2 b,
\end{aligned}
$$

so that

$$
k=4(1-g)+3 a+2 b .
$$

At the same time we have

$$
\begin{aligned}
\mathcal{L}^{3} & =(a \mathcal{V}+2 \mathcal{G})^{3}=12 a+8 \mathcal{G}^{3} \\
& =12 a+8 b+16(1-g)=4 k,
\end{aligned}
$$

proving the equality in (26).

The inequality in $(26)$ we have left to prove is $\mathcal{L}^{3} \geqslant 2 h^{0}(\mathcal{L})-12$. We therefore assume, to get a contradiction, that

$$
\mathcal{L}^{3} \leqslant 2 h^{0}(\mathcal{L})-13
$$

Since the one-dimensional part of the base locus of $|\mathcal{L}|$ can only consist of smooth quartics (with respect to $\mathcal{L}$ ) in the fibers of $f$, we can write, on $S$,

$$
\mathcal{L}_{\mid S} \sim H_{0}+\left(f_{\mid S}\right)^{*} \mathfrak{v} \equiv H_{0}+c F
$$

for some nonnegative integer $c$, where $\mathfrak{v}$ is an effective divisor of degree $c$ on $B$; $F$ denotes the numerical equivalence class of a fiber of $f_{\mid S}: S \rightarrow B$; and $\left|H_{0}\right|$ is the moving part of $\left|\mathcal{L}_{\mid S}\right|$. If the general element $C_{0} \in\left|H_{0}\right|$ were not reduced and irreducible, then by Bertini's theorem $\left|H_{0}\right|$ would be composed with a pencil, whence $H_{0} \equiv m H_{0}^{\prime}$, for some $H_{0}^{\prime} \in \operatorname{Pic} S$ with $H_{0}^{\prime 2}=0$ and $m \geqslant 2$. Now $4=F \cdot \mathcal{L}=F \cdot H_{0}=m F \cdot H_{0}^{\prime}$ implies $m \leqslant 4$. By $(33)$ and the short exact sequence

$$
0 \longrightarrow \mathcal{O}_{T} \longrightarrow \mathcal{O}_{T}(\mathcal{L}) \longrightarrow \mathcal{O}_{S}(\mathcal{L}) \longrightarrow 0
$$

(using the fact that $\mathcal{L}^{3} \geqslant 0$ by $(32)$ ) we get the contradiction

$$
5 \geqslant m+1 \geqslant h^{0}\left(H_{0}\right)=h^{0}\left(\mathcal{O}_{S}(\mathcal{L})\right) \geqslant h^{0}(\mathcal{L})-1 \geqslant 6 .
$$

Therefore $C_{0}$ is a reduced and irreducible curve (possibly singular).

From

$$
0 \longrightarrow \mathcal{O}_{S}\left(f^{*} \mathfrak{v}\right) \longrightarrow \mathcal{O}_{S}(\mathcal{L}) \longrightarrow \mathcal{O}_{C_{0}}(\mathcal{L}) \longrightarrow 0
$$

and (34) we get, using (33),

$$
\begin{aligned}
h^{0}\left(\mathcal{O}_{C_{0}}(\mathcal{L})\right) & \geqslant h^{0}\left(\mathcal{O}_{S}(\mathcal{L})\right)-h^{0}\left(\mathcal{O}_{S}\left(f^{*} \mathfrak{v}\right)\right) \\
& \geqslant h^{0}\left(\mathcal{O}_{T}(\mathcal{L})\right)-h^{0}(B, \mathfrak{v})-1 \\
& \geqslant \frac{1}{2}\left(\mathcal{L}^{3}+13\right)-c-2=\frac{1}{2} \mathcal{L}^{3}-c+\frac{9}{2} .
\end{aligned}
$$

Moreover $\operatorname{deg} \mathcal{O}_{C_{0}}(\mathcal{L})=\mathcal{L}^{2} \cdot(\mathcal{L}-c \mathcal{V})=\mathcal{L}^{3}-c \mathcal{L}^{2} \cdot \mathcal{V}=\mathcal{L}^{3}-4 c$, so that

$$
\operatorname{deg} \mathcal{O}_{C_{0}}(\mathcal{L})-2\left(h^{0}\left(\mathcal{O}_{C_{0}}(\mathcal{L})\right)-1\right) \leqslant \mathcal{L}^{3}-4 c-\left(\mathcal{L}^{3}-2 c+7\right)=-2 c-7<0 .
$$




\section{ON VARIETIES THAT ARE UNIRULED BY LINES}

By Clifford's theorem on singular curves (see the appendix of [EKS88]) we must therefore have

$$
h^{1}\left(\mathcal{O}_{C_{0}}(\mathcal{L})\right)=0 .
$$

Also note that since $T$ is a projective bundle over a smooth curve of genus $g$, we have

$$
h^{0}\left(\mathcal{O}_{T}\right)=1, \quad h^{1}\left(\mathcal{O}_{T}\right)=g, \quad h^{2}\left(\mathcal{O}_{T}\right)=h^{3}\left(\mathcal{O}_{T}\right)=0 .
$$

From Riemann-Roch on $S$ and the fact that $h^{2}\left(\mathcal{O}_{S}\left(f^{*} \mathfrak{v}\right)\right)=h^{0}\left(K_{S}-f_{\mid S}^{*} \mathfrak{v}\right)=0$ (since $F$ is nef with $\left.F \cdot\left(K_{S}-f^{*} \mathfrak{v}\right)=F \cdot\left(K_{S}-c F\right)=-2\right)$ we find

$$
\begin{aligned}
h^{1}\left(\mathcal{O}_{S}\left(f^{*} \mathfrak{v}\right)\right) & =-\chi\left(\mathcal{O}_{S}\left(f^{*} \mathfrak{v}\right)\right)+h^{0}\left(\mathcal{O}_{S}\left(f^{*} \mathfrak{v}\right)\right)+h^{2}\left(\mathcal{O}_{S}\left(f^{*} \mathfrak{v}\right)\right) \\
& =-\frac{1}{2} c F \cdot\left(c F-K_{S}\right)+g-1+h^{0}\left(\mathcal{O}_{S}\left(f^{*} \mathfrak{v}\right)\right) \\
& \leqslant-c+g-1+c+1=g .
\end{aligned}
$$

Combining all of (34)-(39) we find

$$
\begin{aligned}
h^{1}(\mathcal{L}) & \leqslant h^{1}\left(\mathcal{O}_{T}\right)+h^{1}\left(\mathcal{O}_{S}(\mathcal{L})\right) \\
& \leqslant h^{1}\left(\mathcal{O}_{T}\right)+h^{1}\left(\mathcal{O}_{S}\left(f^{*} \mathfrak{v}\right)\right)+h^{1}\left(\mathcal{O}_{C_{0}}(\mathcal{L})\right) \\
& \leqslant g+g+0=2 g
\end{aligned}
$$

together with

$$
h^{2}(\mathcal{L})=h^{3}(\mathcal{L})=0 .
$$

From (28)-(30), (34), (38) and Riemann-Roch on $S$, we get

$$
\begin{aligned}
\chi(\mathcal{L}) & =\chi\left(\mathcal{O}_{S}(\mathcal{L})\right)+\chi\left(\mathcal{O}_{T}\right)=\frac{1}{2} \mathcal{O}_{S}(\mathcal{L}) \cdot\left(\mathcal{O}_{S}(\mathcal{L})-K_{S}\right)+\chi\left(\mathcal{O}_{S}\right)+\chi\left(\mathcal{O}_{T}\right) \\
& =\frac{1}{2}\left(\mathcal{L}^{3}-\mathcal{L}^{2} \cdot\left(K_{T}+\mathcal{L}\right)\right)+\chi\left(\mathcal{O}_{S}\right)+\chi\left(\mathcal{O}_{T}\right)=-\frac{1}{2} \mathcal{L}^{2} \cdot K_{T}+2(1-g) \\
& =-\frac{1}{2}(a \mathcal{V}+2 \mathcal{G})^{2} \cdot(b \mathcal{V}-3 \mathcal{G})+2(1-g)=14(1-g)+6 a+4 b .
\end{aligned}
$$

Comparing with (32) we see that

$$
\mathcal{L}^{3}=2 \chi(\mathcal{L})-12(1-g)
$$

whence, using (40) and (41),

$$
\begin{aligned}
\mathcal{L}^{3} & =2\left(h^{0}(\mathcal{L})-h^{1}(\mathcal{L})+h^{2}(\mathcal{L})-h^{3}(\mathcal{L})\right)-12(1-g) \\
& \geqslant 2\left(h^{0}(\mathcal{L})-2 g\right)-12(1-g)=2 h^{0}(\mathcal{L})-12+8 g \geqslant 2 h^{0}(\mathcal{L})-12,
\end{aligned}
$$

contradicting (33).

This shows that (33) cannot hold, proving (26).

Example 3.4. As in Example 2.5 take $X=\mathbb{P}^{2} \times \mathbb{P}^{1}$ and $\mathcal{H}:=p^{*} \mathcal{O}_{\mathbb{P}^{2}}(2) \otimes q^{*} \mathcal{O}_{\mathbb{P}^{1}}(a)$. Then we have an embedding given by $|\mathcal{H}|$ :

$$
\mathbb{P}^{2} \times \mathbb{P}^{1} \longrightarrow \mathbb{P}^{6(a+1)-1}
$$

A hyperplane section of $X$ in $\mathbb{P}^{6(a+1)-1}$ has equation

$$
\sum_{i, j=0,1,2,0 \leqslant k \leqslant a} l_{i j k} x_{i} x_{j} y_{0}^{k} y_{1}^{a-k}=0,
$$

where $\left(x_{0}: x_{1}: x_{2}\right)$ are the coordinates on $\mathbb{P}^{2}$ and $\left(y_{0}: y_{1}\right)$ are the coordinates on $\mathbb{P}^{1}$ and $l_{i j k}$ are coefficients. The section is degenerate on some Veronese surface $\left(\mathbb{P}^{2}, \mathcal{O}_{\mathbb{P}^{2}}(2)\right)$ if the determinant of the matrix of the coefficients of the $x_{i} x_{j}$ is zero. This determinant is a polynomial of degree $3 a$ in $y_{0}, y_{1}$, hence in general we find $3 a$ distinct zeros. This means that a general hyperplane section 


\section{A. L. Knutsen, C. Novelli And A. Sarti}

has $3 a=\frac{1}{2}(6(a+1)-1-5)$ degenerate fibers, which is the smallest possible number of degenerate fibers for a terminal Veronese fibration as stated in Proposition 3.2.

Proofs of Propositions 0.4 and 2.4. We note that by Proposition 3.2 the only statement left to prove in Proposition 0.4 is a special case of Proposition 2.4.

As in Proposition 2.4 let $(X, \mathcal{H})$ be a pair consisting of a terminal $\mathbb{Q}$-factorial threefold $X$ and a globally generated, big line bundle $\mathcal{H}$ on $X$, with $d:=d(X, \mathcal{H})$ and $n:=n(X, \mathcal{H})$.

Assume that a $\sharp$-minimal model $\left(X^{\sharp}, \mathcal{H}^{\sharp}\right)$ is of type (II) in Theorem $1.2(\mathrm{v})$, i.e. a terminal Veronese fibration over a smooth curve $B$ of genus $g$.

Let $f: S \rightarrow S^{\sharp}$ be as in Theorem 1.2(iii). We have $n^{\sharp}:=\operatorname{dim}\left|\mathcal{H}^{\sharp}\right| \geqslant \operatorname{dim}|\mathcal{H}|=n$ by Lemma 1.5(a). By Proposition 3.2, $S^{\sharp}$ is fibered over $B$ with general fiber a smooth quartic and $k \geqslant \frac{1}{2}\left(n^{\sharp}-5\right)$ fibers being the union of two rational curves, intersecting in one point, that are both $(-1)$-curves. Therefore

$$
K_{S^{\sharp}}^{2}=8(1-g)-k \leqslant 8(1-g)-\frac{1}{2}\left(n^{\sharp}-5\right) \leqslant 8(1-g)-\frac{1}{2}(n-5) .
$$

We want to show that $d \geqslant 2 n-10$. Assume, to get a contradiction, that

$$
d \leqslant 2 n-11 \text {. }
$$

Note that $\rho:=\rho(X, \mathcal{H})=\frac{2}{3}$, so we can apply Lemma 1.5(b). Let $l$ be the total number of irreducible curves contracted by $f$. Then $K_{S}^{2}=K_{S^{\sharp}}^{2}-l$. Pick any smooth irreducible curve $D \in$ $\left|\mathcal{O}_{S}(\mathcal{H})\right|$. Then by $(3),(10)$ and $(42)$ we have

$$
\begin{aligned}
0 & \leqslant 4 l+\left(D+2 K_{S}\right)^{2}=4 l+4 K_{S}^{2}+4 K_{S} \cdot D+D^{2} \\
& \leqslant 4 l+4\left(8(1-g)-\frac{1}{2}(n-5)-l\right)+4(d-2 n+2)+d \\
& =32(1-g)-2(n-5)+4 d-8 n+8+d \\
& =32(1-g)+5 d-10 n+18 \leqslant 5 d-10 n+50=5(d-2 n+10),
\end{aligned}
$$

contradicting (43).

We have therefore proved that $d \geqslant 2 n-10$ and this finishes the proofs of Propositions 0.4 and 2.4 .

\section{Final remarks}

To conclude, we remark that a closer look at the proofs of Propositions 0.4 and 2.4 shows that, if we assume only $d<2 n-4$ instead of (43), we get $g=0$ as the only possibility. This shows that the following holds:

a three-dimensional terminal Veronese fibration over a smooth curve of genus $g>0$ must satisfy $d \geqslant 2 n-4$.

Consequently we also have the following:

if a pair $(X, \mathcal{H})$ consisting of a terminal $\mathbb{Q}$-factorial threefold $X$ and a globally generated, big line bundle $\mathcal{H}$ on $X$ has a $\sharp$-minimal model being of type (II) in Theorem 1.2(v) over a smooth curve of genus $g>0$, then $d \geqslant 2 n-4$.

If now $\left(X^{\sharp}, \mathcal{H}^{\sharp}\right)$ is a $\sharp$-minimal model of a pair $(X, \mathcal{H})$ consisting of a terminal $\mathbb{Q}$-factorial threefold $X$ and a globally generated big line bundle $\mathcal{H}$, then $\mathcal{H}^{\sharp}$ is still big and nef by Lemma 1.5 (a), so that $h^{1}\left(\mathcal{O}_{X}\right)=h^{1}\left(\mathcal{O}_{S}\right)=h^{1}\left(\mathcal{O}_{S^{\sharp}}\right)=h^{1}\left(\mathcal{O}_{X^{\sharp}}\right)$. We have seen that this is zero if $X^{\sharp}$ is of type (I) in Theorem 1.2(v) and equal to $g$, the genus of $B$, if $X^{\sharp}$ is of type (II) in Theorem $1.2(\mathrm{v})$.

We have therefore obtained an improvement of [Mel02, Theorem 5.8] (cf. Theorem 1.2(v)). 


\section{ON VARIETIES THAT ARE UNIRULED BY LINES}

Proposition 4.1. Let $(X, \mathcal{H})$ be a pair consisting of a terminal $\mathbb{Q}$-factorial threefold $X$ and a globally generated big line bundle $\mathcal{H}$ on $X$. Set $d:=\mathcal{H}^{3}$ and $n:=h^{0}(X, \mathcal{H})-1$.

If $d<2 n-10$ (respectively $d<2 n-4$ and $h^{1}\left(\mathcal{O}_{X}\right)>0$ ), then $\left(X^{\sharp}, \mathcal{H}^{\sharp}\right)$ is of one of the types (i)-(iv) (respectively (ii)-(iv)) below:

(i) $\left(\mathbb{P}^{3}, \mathcal{O}_{\mathbb{P}^{3}}(3)\right)($ with $(d, n)=(27,19))$;

(ii) a quadric bundle with at most $c A_{1}$ singularities of type $f=x^{2}+y^{2}+z^{2}+t^{k}$, for $k \geqslant 2$, and $\mathcal{H}_{\mid F}^{\sharp} \sim \mathcal{O}_{F}(1)$ for every fiber $F$;

(iii) $(\mathbb{P}(E), \mathcal{O}(1))$ where $E$ is a rank 3 vector bundle over a smooth curve;

(iv) $(\mathbb{P}(E), \mathcal{O}(1))$ where $E$ is a rank 2 vector bundle over a surface of negative Kodaira dimension.

Consequently we have the following slight improvement of Theorem 0.1 and Corollary 0.2.

Corollary 4.2. Let $(X, \mathcal{H})$ be a pair consisting of a reduced and irreducible $k$-dimensional variety $X, k \geqslant 3$, and a globally generated line bundle $\mathcal{H}$ on $X$. Set $d:=\mathcal{H}^{k}$ and $n=h^{0}(X, \mathcal{H})-1$.

If $h^{1}\left(\mathcal{O}_{\tilde{X}}\right)>0$ for a resolution of singularities $\tilde{X}$ of $X$ and $d<2(n-k)+2$, then $X$ is uniruled of $\mathcal{H}$-degree one.

Proof. In the proof of Theorem 0.1, use Proposition 4.1 in place of Theorem 1.2(v). Then, in the proof of Corollary 0.2 , note that $h^{1}\left(\mathcal{O}_{X_{i}}\right)=h^{1}\left(\mathcal{O}_{X_{i-1}}\right)$ as $\mathcal{H}_{i}$ is big and nef.

\section{ACKNOWLEDGEMENTS}

We are indebted to M. Mella for suggesting the problem and for many helpful discussions. We were introduced to the topic during the wonderful school Pragmatic 2002 in Catania, and it is a great pleasure to thank all the participants, as well as the organizer A. Ragusa. We also thank M. Andreatta, R. Pignatelli, W. Barth and K. Ranestad for useful comments.

We would also like to thank the referees for pointing out a mistake in the first version and for suggestions that have made the paper clearer and easier to read.

\section{REFERENCES}

Ba75 W. Barth, Submanifolds of low codimension in projective space, in Proc. Internat. Congress of Mathematicians, Vancouver, BC, 1974, vol. 1 (Canadian Mathematics Congress, Montreal, 1975), 409-413.

CF93 F. Campana and H. Flenner, Projective threefolds containing a smooth rational surface with ample normal bundle, J. reine angew. Math. 440 (1993), 77-98.

EKS88 D. Eisenbud, J. Koh and M. Stillman, Determinantal equations for curves of high degree, with an appendix by J. Harris, Amer. J. Math. 110 (1988), 513-539.

Fuj75 T. Fujita, On the structure of polarized varieties with $\Delta$-genera zero, J. Fac. Sci. Univ. Tokyo Sect. IA Math. 22 (1975), 103-115.

Fuj77 T. Fujita, Defining equations for certain types of polarized varieties, in Complex analysis and algebraic geometry, eds W. L. Baily and T. Shioda (Iwanami Shoten, Tokyo, 1977), 165-173.

Fuj80 T. Fujita, On the structure of polarized manifolds with total deficiency one I, J. Math. Soc. Japan 32 (1980), 709-725.

Fuj81 T. Fujita, On the structure of polarized manifolds with total deficiency one II, J. Math. Soc. Japan 33 (1981), 415-434.

Fuj84 T. Fujita, On the structure of polarized manifolds with total deficiency one III, J. Math. Soc. Japan 36 (1984), 75-89. 


\section{ON VARIETIES THAT ARE UNIRULED BY LINES}

GLM04 L. Giraldo, A. F. Lopez and R. Munoz, On the existence of Enriques-Fano threefolds of index greater than one, J. Algebraic Geom. 13 (2004), 143-166.

Har74 R. Hartshorne, Varieties of small codimension in projective space, Bull. Amer. Math. Soc. 80 (1974), 1017-1032.

Hor83 T. Horowitz, Varieties of low $\Delta$-genus, Duke Math. J. 50 (1983), 667-683.

Ion85 P. Ionescu, On varieties whose degree is small with respect to codimension, Math. Ann. 271 (1985), 339-348.

Isk77 V. A. Iskovskih, Fano threefolds I, Izv. Akad. Nauk SSSR Ser. Mat. 41 (1977), 516-562, 717.

Kol96 J. Kollar, Rational curves on algebraic varieties, Ergebnisse der Mathematik und ihrer Grenzgebiete, vol. 32 (Springer, Berlin, 1996).

Lvo92a S. L'vosky, Extension of projective varieties and deformations I, Michigan Math. J. 39 (1992), $41-51$.

Lvo92b S. L'vosky, Extension of projective varieties and deformations II, Michigan Math. J. 39 (1992), $65-70$.

Mel97 M. Mella, Adjunction theory on terminal varieties, in Complex analysis and geometry, Trento, 1995, Pitman Research Notes in Mathematics, vol. 366 (Longman, Harlow, 1997), 153-164.

Mel02 M. Mella, \#-minimal models of uniruled 3-folds, Math. Z. 242 (2002), 687-707.

Miy88 Y. Miyaoka, On the Kodaira dimension of minimal threefolds, Math. Ann. 281 (1988), 325-332.

Rei86 M. Reid, Surfaces of small degree, Math. Ann. 275 (1986), 71-80.

Xia86 G. Xiao, Algebraic surfaces with high canonical degree, Math. Ann. 274 (1986), 473-483.

Zak91 F. L. Zak, Some properties of dual varieties and their applications in projective geometry, in Algebraic geometry, Chicago, IL, 1989, Lecture Notes in Mathematics, vol. 479 (Springer, Berlin, 1991), 273-280.

Andreas L. Knutsen andreas.knutsen@math.uio.no, knutsen@mat.uniroma3.it

Department of Mathematics, University of Oslo, PO Box 1053, Blindern, NO-0316 Oslo, Norway.

Current address: Dipartimento di Matematica, Università di Roma Tre, Largo San Leonardo Murialdo 1, 00146 Roma, Italy

Carla Novelli novelli@science.unitn.it

Dipartimento di Matematica, Università degli Studi di Trento, via Sommarive 14, 38050 Povo (TN), Italy

Alessandra Sarti sarti@mathematik.uni-mainz.de Institut für Mathematik, Johannes-Gutenberg-Universität Mainz, Staudingerweg 9, 55099 Mainz, Germany 